\title{
Zur Stereochemie einfacher Moleküle, einfacher Ionen und von Atomgruppen in größeren Molekülen ${ }^{1}$
}

\author{
Von Burckhardt Helferich* \\ (Z. Naturforschg. 1, 666-670 [1916]; eingegangen am 25. Juli 1946)
}

\begin{abstract}
Der Zusammenhang zwischen räumlicher Anordnung und Valenzelektronenschale des Zentralatoms wird für Atomanordnungen der allgemeinen Formel $\Lambda \mathrm{X}_{2}$ und $\mathrm{AX}_{3}$ in zwei einfachen Regeln zusammengefaßt. Es wird ein Vorschlag gemacht, diese Regeln auch auf Gruppierungen $\mathrm{A} \mathrm{X}_{4}$ auszudehnen.
\end{abstract}

Cchon mehrfach ist in einzelnen Fällen darauf Shingewiesen worden, daß für die räumliche Lagerung von drei Liganden um ein Zentralatom die Existenz eines unbeteiligten Elektronenpaares von Bedeutung ist. So schreibt V. M. Goldschmidt ${ }^{2}$ im Anschluß an eine Arbeit von $\mathrm{Za}$ chariasen ${ }^{3}$; ,In allen diesen Fällen (pyramidaler Bau von $\mathrm{AX}_{3}$ ) handelt es sich, wie man sieht, bei den Partikeln A, falls man sie formal als Ionen auffaßt, nicht um edelgasähnliche Ionen, sondern um Ionen mit einem Elektronenpaar außerhalb der Elektronenhülle“. Bei K. u. U. H of mann ${ }^{4}$ findet man: ,Diese Gestalt (Pyramide) entspricht einem tetraedrischen Durchdringungskomplex mit Edelgaskonfiguration, dem der vierte Ligand fehlt. Tatsächlich haben ja auch alle Zentralatome dieser Liganden noch ein unbetätigtes Elektronenpaar."Eugen M ülle r schreibt ${ }^{5}$ von den Sulfoxyden: „...als vierter Substituent fungiert vermutlich das noch einsame Elektronenpaar des Schwefelatoms."

In der vorliegenden Arbeit ist dieser Gedanke systematisch auf eine größere Anzahl von Atomgruppen $\mathrm{AX}_{2}$ und $\mathrm{AX}_{3}$ ausgedehnt und in zwei allgemeine Regeln zusammengefaßt worden. Diese Regeln scheinen so allgemeine Gültigkeit zu haben, daß sie auch Vorhersagen gestatten über den räumlichen Bau entsprechender Atomgruppen, wenn die experimentellen Grundlagen dazu noch nicht ausreichen.

A. Der räumliche Bau der Atomgruppierung $A X_{2}$ kann entweder linear sein $\mathrm{X}-\mathrm{A}-\mathrm{X}$ oder

* z. Zt. in Weilburg (Lahn) und Bonn.

${ }^{1}$ Der Inhalt der Arbeit ist im Colloquium des Chemischen Instituts der Universität Bonn am 21. Mai $1944^{\circ}$ vorgetragen worden.

2 K. F r e ud en be r g. Stereochemie. Verlag Franz Deuticke, 1933, S. 51.

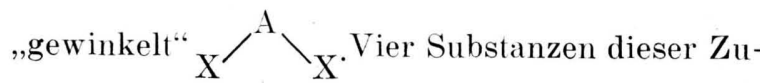
sammensetzung, deren räumlicher Bau durch physikalische Methoden gesichert ist, sind die Moleküle $\mathrm{CO}_{2}, \mathrm{SO}_{2}, \mathrm{OH}_{2}, \mathrm{NO}_{2}$.

1. $\mathrm{CO}_{2}$ ist linear gebaut und entspricht der Formulierung $\mathrm{O}=\mathrm{C}=\mathrm{O}$. Am - zentralen - Kohlenstoffatom sind keine unbetätigten Elektronen vorhanden.

2. $\mathrm{SO}_{2}$ ist ,gewinkelt": $\quad \overline{\mathrm{O}}^{\overline{\mathrm{S}}} \overline{\mathrm{O} \mid}$. Die Formulierung, einerlei wie sie gewählt wird, beläßt stets ein unbeteiligtes Elektronenpaar (im weiteren auch mit u. Ep. bezeichnet) am Schwefel.

3. Dàs Wassermolekül ist ebenfalls gewinkelt: $\mathrm{H}^{\prime} \mathrm{O}_{\mathrm{H}^{\prime}}$ An seinem Zentralatom, dem Sauerstoff, sind zwei Elektronenpaare unbeschäftigt.

4. Nach neueren Ergebnissen ${ }^{6}$ ist auch das $\mathrm{NO}_{2}$ gewinkelt. Der zentrale Stickstoff dieses Moleküls (Radikals) trägt ein unbeteiligtes Elektron:

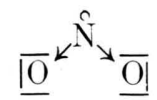

Die erste Regel, die aus diesen Beispielen abgeleitet sei, kann in die folgenden Worte gefaßt werden:

I. Atomgruppierungen der allgemeinen Formel $\mathrm{AX}_{\text {, }}$ (X untereinander gleich oder verschieden) haben linearen Bau, wenn am Zentralatom keine unbeteiligten Elektronen vorhanden sind. Sie sind "gewinkelt", wenn das Zentralatom A ein oder zwei unbeteiligte Elektronenpaare - oder ein ein-

3 Amer. chem. Soc. 53, 2123 [1931].

4 Anorganische Chemie. Verlag Friedr. Vieweg. Braunschweig, 9. Aufl., 1941, S. 723.

5 Neuere Anschauungen der Organischen Chemie. Verlag J. Springer, Berlin 1940.

${ }^{*}$ Chem. Zbl. 1933 I, 1308. 
zelnes, nicht an den Bindungen beteiligtes Elektron - trägt.

Diese Regel sei auf die folgenden weiteren Fälle angewandt:

\section{Zentralatom $\mathrm{A}=$ Kohlenstoff.}

Ebenso wie $\mathrm{CO}_{2}$, so sind auch $\mathrm{CS}_{2}$ und $\mathrm{SCO}$ linear gebaut. In den Allenen ist die Gruppe $\mathrm{C}=\mathrm{C}=\mathrm{C} \backslash$ linear, ebenso die Gruppe $\mathrm{H}-\mathrm{C} \equiv \mathrm{C}-$ in dem Acetylen und seinen Derivaten $-\mathrm{C}-\mathrm{C} \equiv \mathrm{C}-$. Blausäure ist linear, $\mathrm{H}-\mathrm{C} \equiv \mathrm{N} \mid$, ebenso die Nitrile $-\mathrm{C}-\mathrm{C} \equiv \mathrm{N} \mid$, als Derivate der Blausäure. Die Cyansäure $\mid \mathrm{N} \equiv \mathrm{C}-\mathrm{O}-$, ihr Ion $|\mathrm{N} \equiv \mathrm{C}-\overline{\mathrm{O}}|(-)$ und ihre N-bzw. C-Derivate haben die Gruppe $\mathrm{NCO}$ in linearer Lage. Das gleiche gilt für den Rhodanwasserstoff und seine Abkömmlinge. Auch das Cyanamid-Ion $/ \mathrm{N}=\mathrm{C}=\mathrm{N}\rangle(-\rightarrow)$ ist linear gebaut und damit die Gruppe $\mathrm{NCN}^{\prime}$ auch in ihren Derivaten.

\section{Zentralatom $\mathrm{A}=$ Stickstoff.}

Linear sind $\mathrm{N}_{2} \mathrm{O}$ und das Ion der Stickstoffwasserstoffsäure $\mathrm{N}_{3}$. Ihre Formelbilder $\langle\mathrm{N}=\mathrm{N}=\mathrm{O}\rangle$ und $\angle \mathrm{N}=\mathrm{N}=\mathrm{N}\rangle(-)$ tragen am Z Zentralatom keine unbeteiligten Elektronen.

„Gewinkelt" haften dagegen die Nachbarn am Stickstoff in den Oximen $\searrow_{\mathrm{C}}=\overline{\mathrm{N}}_{\mathrm{OH}}$, den Azoverbindungen ${ }^{\mathrm{R}} \searrow_{\mathrm{N}}=\overline{\mathrm{N}}_{\backslash_{\mathrm{R}}}$ bzw. ${ }_{\mathrm{R}} / \overline{\mathrm{N}}=\overline{\mathrm{N}}_{\searrow_{\mathrm{R}}}$

und den Azoxyverbindungen $\mathrm{R} / \overline{\mathrm{N}}=\mathrm{N} \lambda \frac{\overline{\mathrm{Ol}}}{\mathrm{R}}$, ebenso in dem Nitrit-Ion $\underline{\mid \bar{O}} \kappa^{\overline{\mathrm{N}}_{\searrow}}{ }^{(-)}{ }^{(-)}$und damit in der salpetrigen Säure und ihren Derivaten (Estern) und den Nitrosoverbindungen $-\mathrm{C}-\mathrm{N}^{\pi} \stackrel{\overline{\mathrm{Ol}}}{ }$. In allen diesen Fällen trägt der zentrale Stickstoff ein unbeteiligtes Elektronenpaar.

\section{Zentralatom A = Sauerstoff oder Schwefel.}

Die „Winkelung“ des Wassers (s. o.) findet man in allen seinen Derivaten wieder, also in den $\mathrm{Al}$ koholen, den Estern, den Äthern, allen Hydroxylgruppen, dem Wasserstoffperoxyd und seinen
Derivaten. Stets sind am zentralen Sauerstoff zwei u. Ep. vorhanden.

Das gleiche gilt für den Schwefelwasserstoff und seine Derivate und läßt sich auch auf die entsprechenden Verbindungen des Selens und Tellurs übertragen.

\section{Zentralatom $\mathrm{A}=$ Wasserstoff.}

Bei „Wasserstoffbrücken" ist der Wasserstoff mit zwei nächsten Nachbarn - wenn auch vielleicht nicht ganz gleichmäßig - verknüpft. Selbst trägt er sicher keine u. Ep. Dementsprechend ist das Ion $\mathrm{F}-\mathrm{H}-\mathrm{F}^{(-)}$linear gebaut. Im gleichen Sinne sind durch Wasserstoffbrücken entstandene Ringe aufzufassen. Der Ring des Salicylaldehyds ist ein $F \ddot{u n f}$-Eck, entsprechend der Formulierung, kein Sechs-Eck:<smiles>O[C@@H]1[CH]CCCC1</smiles>

Die Anordnung der beiden Sauerstoffatome um den Brückenwasserstoff ist - annähernd? linear.

B. Der räumliche Bau der Atomgruppierung $\mathrm{A} \mathrm{X}_{3}$ (X untereinander gleich oder verschieden) kann eben sein (alle vier Atome in einer Ebene) oder pyramidal, mit $\mathrm{A}$ an der Spitze einer dreiseitigen Pyramide. Beispiele für den ersten Fall, ebene Anordnung, sind $\mathrm{BF}_{3}$ und $\mathrm{CO}_{3}(--)$, für den zweiten Fall, pyramidale Anordnung, $\mathrm{PF}_{3}$ und $\mathrm{ClO}_{3}{ }^{(-)}$. Im ersten Fall tragen die Zentralatome B und $\mathrm{C}$ kein u. Ep.

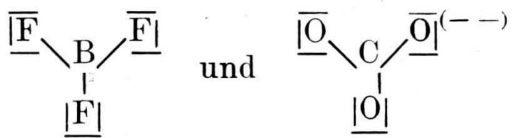

Im zweiten Fall dagegen ist am Phosphor wie am Chlor je ein u. Ep. vorhanden.

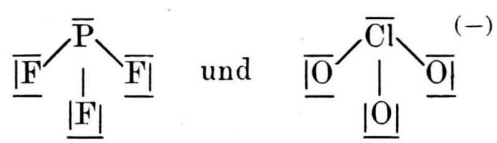

Aus diesen Beispielen sei als zweite Regel formuliert :

II. Atomgruppierungen der allgemeinen Formel $\mathrm{AX}_{3}$ haben ebene Anordnung (X untereinander gleich oder verschieden), wenn das Zentralatom A keine unbeteiligten Elektronen trägt. Es 
bildet sich pyramidale Anordnung, mit A an der - Spitze einer dreiseitigen Pyramide, aus, wenn das Zentralatom A unbeteiligte Elektronen - ein unbeteiligtes Elektronenpaar - trägt.

Es seien folgende weitere Beispiele aufgezählt:

\section{Zentralatom $\mathrm{A}=$ Kohlenstoff.}

Ebene Gruppierung von drei Liganden um den Kohlenstoff ist vorhanden in der Kohlensäure und ihren Estern,<smiles>[13CH3]OC(=O)[18OH]</smiles>

im Harnstoff und seinen Derivaten $\mathrm{O}=\mathrm{C}>_{\mathrm{NH}_{2}}^{\mathrm{NH}_{2}}$, im Phosgen $\mathrm{O}=\mathrm{C} \nearrow_{\mathrm{Cl}}^{\mathrm{Cl}}$, in der Carbaminsäure und ihren Derivaten. Ebenso ist es bei dem doppelt gebundenen Kohlenstoff in den Äthylenen ${ }_{\mathrm{R}}^{\mathrm{R}} \backslash \mathrm{C}=\mathrm{C}$ 〈' in den Aldehyden ${ }_{\mathrm{R}}^{\mathrm{H}} \searrow_{\mathrm{C}=\mathrm{O}}$, den Ketonen, den Säurechloriden

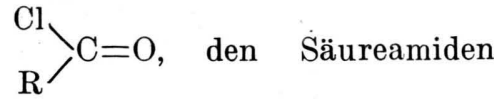
$\mathrm{H}_{2} \mathrm{~N}_{\triangle} \mathrm{C}=\mathrm{O}$. Auch der aromatische Kohlenstoff des Benzols ${ }_{\mathrm{C}}^{\mathrm{H}} \mathrm{C}_{0}-\mathrm{C}$ ist hierher zu rechnen, da er mit den drei nächsten, mit ihm verknüpften Nachbarn in einer Ebene liegt. Das „vierte“ Elektron wird für den aromatischen Zustand gebraucht, ist also nicht unbeteiligt.

\section{Zentralatom $\mathrm{A}=$ Stickstoff.}

Ebene Anordnung um den Stickstoff ist nachgewiesen im Nitrat-Ion

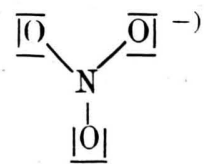

und damit in der Salpetersäure und ihren Estern anzunehmen, ebenso in der Nitrogruppe,

im Pyridin- $N$-oxyd<smiles>CC(C)(C)[N+](=O)[O-]</smiles><smiles>CI=C(C)C</smiles>

und vermutlich auch im Pyrrol und seinen $N$-Derivaten, bei dem die zwei Elektronen des $\mathrm{N}$ zum aromatischen Zustand

verbraucht sind.<smiles>C=CN(C=C)C=C</smiles>

Andere N-Verbindungen mit pyramidalem Bau sind weiter unten angeführt. In den bisher angegebenen ist am Stickstoff kein u. Ep. anzunehmen.

3. Zentralatom $\mathrm{A}=$ Bor.

Ebene Lage ist erwiesen für die Moleküle $\mathrm{BH}_{3}$, $\mathrm{BF}_{3}$ und für das Ion $\mathrm{BO}_{3}(---)$. U. Ep. sind am Bor in diesen Substanzen nicht vorhanden.

4. Zentralatom $\mathrm{A}=$ Zinn.

Vielleicht darf der folgende Fall angeführt werden: Salze des Methyl-äthyl-propyl-zinn-hydroxyds

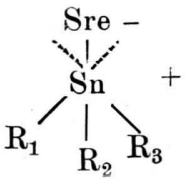

mit optisch aktiven Säuren sind optisch aktiv, also tetraedrisch gebaut. Das Kation aber racemisiert sich sehr rasch, weil in ihm am Zinn kein u. Ep. vorhanden ist, die Tetraderform also verloren geht und dem ebenen Bau Platz macht.

\section{Zentralatom $\mathrm{A}=$ Element der 5. Gruppe $(\mathrm{N}, \mathrm{P}$, $\mathrm{As}, \mathrm{Sb})$.}

Für die Wasserstoffverbindungen dieser Elemente von der Formel<smiles>[H][1H]</smiles>

ist pyramidaler Bau nachgewiesen. Am Zentralatom sitzt, als „vierter Ligand“, ein u.Ep. Das gleiche ist der Fall für die Halogenverbindungen der allgemeinen Formel $\mathrm{A} \mathrm{Hlg}_{3}$.

Für alle primären, sekundären und tertiären Amine, weiter für die Säureamide (um das NAtom) darf ebenfalls pyramidaler Bau angenommen werden. Für das Ion<smiles>O[AsH2](O)O</smiles> 
ist der pyramidale Bau erwiesen und damit auch für die analogen Ionen der anderen Elemente dieser Gruppe. Auch in diesen Fällen ist am Zentralatom ein u. Ep. vorhanden.

\section{Zentralatom $\mathrm{A}=$ Element der 6. Gruppe.}

a) Schwefel.

Das $\overline{\mathrm{SO}}_{3}$-Molekül ist eben gebaut. Das Zentralatom, der Schwefel, trägt in ihm kein u. Ep.

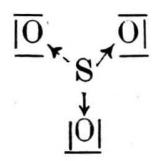

Dagegen ist das Sulfit-Ion pyramidal.

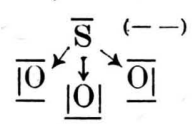

Einerlei, wie man es formuliert, es bleibt am Schwefel stets ein u. Ep., das nirgends anders untergebracht werden kann. Das gleiche ist der Fall in den Sulfonium-Ionen $\mathrm{SR}_{3}{ }^{(+)}$<smiles>[R][Sb]([R])([R])[K]</smiles>

den Sulfoxyden

den'Sulfimiden

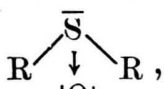

$$
\begin{aligned}
& \text { |O| }
\end{aligned}
$$

$$
\mathrm{R}_{\mathrm{N}}^{\mathrm{S}_{\mathrm{S}}^{\mathrm{S}_{\mathrm{N}}}}{ }_{\mathrm{R}}
$$

und den Sulfinsäureestern

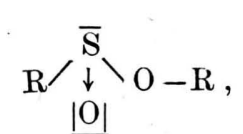

deren Bau durch Spaltung in optisch aktive Komponenten als pyramidal (tetraedrisch) bewiesen ist.

b) Sauerstoff.

Eine pyramidale Lage ist_auch für das Hydronium-Ion

$$
\left.{ }_{\mathrm{H}}^{\mathrm{O}}\right|_{\mathrm{H}} ^{(+)}
$$

anzunehmen und für seine ,organischen“ Derivate. Als solche hat man die Anlagerungsprodukte eines Protons an Alkohole, Ester, Ather, Acetale u. a. anzusehen. Der pyramidale Bau dieser Substanzen und damit die Möglichkeit ihrer Asymmetrie ist - trotz ihrer Unbeständigkeit - vielleicht von Bedeutung bei der fermentativen Spaltung von Estern, Acetalen, Glykosiden usw.

c) Auf die analogen Verbindungen des Selens und des Tellurs wird man die Ergebnisse an den Schwefelverbindungen unbedenklich übertragen dürfen.

\section{Zentralatom $\mathrm{A}=$ Element der 7. Gruppe.}

Pyramidale Struktur ist nachgewiesen für die Ionen $\mathrm{ClO}_{3}(-)$ (s. o.) und $\mathrm{BrO}_{3}^{(-)}$. Auch in ihnen trägt das Zentralatom ein $\mathrm{u}$. Ep.

Die beiden Regeln haben nicht nur für einfache Moleküle oder Ionen Bedeutung. Ihre Anwendung auf Atomgruppen in größeren Molekülen kann für deren räumlichen Bau brauchbare Anhaltspunkte bieten. Dies ist z. B. der Fall in Peptiden, in denen die drei Nachbarn am Säureamid-Kohlenstoff in einer Ebene liegen, während am Säureamid-Stickstoff pyramidale Lagerung anzunehmen ist. Die ebene Anordnung der Kohlenstoffatome in kondensierten aromatischen Ringsystemen, bis hinauf zum Graphit, kann ebenfalls aus der ebenen Lage des aromatischen Kohlenstoffs mit seinen drei nächsten Nachbarn abgeleitet werden, ebenso die Lage der Kohlenstoffatome und der Stickstoffatome in einer Ebene in den Porphinen und Phthalocyaninen. An allen diese Atomen sind die Elektronen zur Bindung der Nachbarn oder für den aromatischen Zustand verbraucht, keine unbeteiligten, Platz beanspruchenden Elektronen mehr vorhanden.

Eine völlige Starrheit der Lage dieser Atomgruppierungen kann nicht behauptet werden. Außerhalb der Gruppe liegende Kräfte, des Moleküls oder des Kristalls, können - in einzelnen Fällen deformierend wirken.

Aber die Regeln fassen ein großes experimentelles Material in für den Chemiker einfacher Form zusammen und können mit erheblicher Wạhrscheinlichkeit auf neue Fälle angewandt werden. Sie sind einfacher und umfassender als die von $\mathrm{Z}$ a c h a ri a s e ${ }^{3}$ aufgestellten Formeln. $\mathrm{Ob}$ und wieweit diese Anschauungen und Regeln über das Formale hinaus eine physikalische Bedeutung haben, soll hier nicht erörtert werden.

C. Die Gedanken, die zu den beiden Regeln I und II geführt haben, sollen im folgenden noch 
auf einige Beispiele des räumlichen Baus von Komplexen der Formel $\mathrm{A} \mathrm{X}_{4}$ ausgedehnt werden. Diese Komplexe können entweder eben:<smiles>[X][X]([X])([X])[X]</smiles>

oder tetraedrisch gebaut sein.

Die tetraedrische Anordnung ist die mehr symmetrische und wird sich ausbilden, wenn keine Gegenkräfte vorhanden sind. Diese können in der Raumerfüllung des Zentralatoms und der vier Liganden liegen. Sie können aber vielleicht auch — ähnlich wie bei den oben erörterten Fällen I und II - in dem Vorhandensein von unbeteiligten Elektronen in der äußeren Hülle des Zentralatoms zu suchen sein, z. B. in den folgenden Fällen:

In dem Salz $\left[\mathrm{J} \mathrm{F}_{4}\right] \mathrm{K}$ ist das Ion $\left[\mathrm{J} \mathrm{F}_{4}\right]$ - eben gebaut. Von den 8 Elektronen des Jodions sind vier zur Bindung der 4 Fluor verbraucht. 4 Elektronen, d. h. zwei Paare, sind unbeteiligt, können zwei Stellen einer oktaedrischen Anordnung um das Jod besetzen und damit die 4 Fluor in die Ebene der vier anderen Stellen drängen.

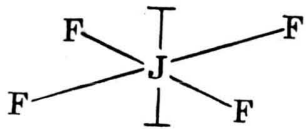

Komplexe der zweiwertigen Ionen vom Nickel, Kupfer, Palladium, Platin und vom dreiwertigen Gold, wie $\left[\mathrm{Ni}(\mathrm{CN})_{4}\right]^{--},\left[\mathrm{Cu}(\mathrm{CN})_{4}\right]^{--}$,
$\left[\mathrm{Pd} \mathrm{Cl}_{4}\right]^{--},\left[\mathrm{Pt} \mathrm{Cl}_{4}\right]^{--},\left[\mathrm{Au} \mathrm{Br}{ }_{4}\right]^{---}$, sind eben (plan) gebaut. In allen Fällen liegt beim ZentralIon keine abgeschlossene Edelgasschale vor. Beim zweiwertigen Kupfer sind es $2+6+9$, bei den anderen $2+6+8$ Elektronen. Vielleicht hat man sich vorzustellen, daß von dieser nicht abgeschlossenen Schale Elektronen oder Elektronenpaare die Rolle der u. Ep. übernehmen, in einer oktaedrischen Anordnung um das Zentralatom an gegenüberliegenden Stellen zwei Plätze besetzen und damit für die vier anderen Liganden nur vier Plätze in einer Ebene übriglassen.

Im Gegensatz dazu haben einwertiges Kupfer und einwertiges Silber als Ionen in Komplexen eine abgeschlossene Edelgasschale $(2+6+10)$. Daraufhin ist der Komplex $\mathrm{NC}-\mathrm{Ag}-\mathrm{CN}(-)$ linear, der Komplex $\left[\mathrm{Cu}(\mathrm{CN})_{4}\right]^{(--)}$tetraedrisch gebaut. Es stehen aus der abgeschlossenen Elektronenschale des Zentral-Ions keine unbeteiligten Elektronen für die Beanspruchung von Plätzen einer oktaedrischen Anordnung zur Verfügung.

Eine aus diesen Beispielen abgeleitete dritte Regel könnte folgendermaßen lauten:

III. Komplexe $\mathrm{AX}_{4}$ sind tetraedrisch gebaut, wenn das Zentral-Ion (Zentralatom) A keine unbeteiligten Elektronen in seiner äußeren Schale enthält und diese Schale edelgasähnlich abgeschlossen ist. Sie sind eben gebaut, wenn das Zentral-Ion (Zentralatom) in einer nicht edelgasähnlich abgeschlossenen äußeren Schale unbeteiligte Elektronen enthält.

$\mathrm{Ob}$ auch diese Regel in ähnlichem Umfang wie die Regeln I und II gilt, muß erst die weitere Prüfung ergeben. 